\title{
KH 15D: GRADUAL OCCULTATION OF A PRE-MAIN-SEQUENCE BINARY
}

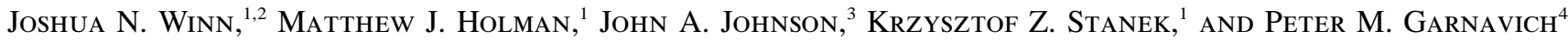 \\ Received 2003 December 17; accepted 2004 January 20; published 2004 February 12
}

\begin{abstract}
We propose that the extraordinary "winking star" $\mathrm{KH} 15 \mathrm{D}$ is an eccentric pre-main-sequence binary that is gradually being occulted by an opaque screen. This model accounts for the periodicity, depth, duration, and rate of growth of the modern eclipses; the historical light curve from photographic plates; and the existing radial velocity measurements. It also explains the rebrightening events that were previously observed during eclipses and the subsequent disappearance of these events. We predict the future evolution of the system and its full radial velocity curve. Given the small velocity of the occulting screen relative to the center of mass of the binary, the screen is probably associated with the binary and may be the edge of a precessing circumbinary disk.
\end{abstract}

Subject headings: circumstellar matter — open clusters and associations: individual (NGC 2264) stars: individual (KH 15D) - stars: pre-main-sequence -

\section{INTRODUCTION}

While monitoring stars in the young cluster NGC 2264, Kearns \& Herbst (1998) noted that star 15 in field D of their sample had a bizarre light curve. This object, known as KH 15D, undergoes periodic eclipses $(P=48.35$ days) that are remarkable for their depth ( $3.5 \mathrm{mag}$ ) and duration (currently $\approx 24$ days). There is consensus that the eclipses are caused by circumstellar material but not on the composition or spatial distribution of that material. Theories include an edge-on circumstellar disk (Hamilton et al. 2001; Herbst et al. 2002; Agol et al. 2004; Winn et al. 2003), an orbiting vortex of solid particles (Barge \& Viton 2003), and an asymmetric common envelope (Grinin \& Tambovtseva 2002). The system has attracted the attention of numerous observers because the fortuitous alignment may allow unique studies of circumstellar (or even protoplanetary) processes and because the occulting edge can be used as a "natural coronagraph" to map out the environment of the underlying T Tauri star (Hamilton et al. 2003; Agol et al. 2004; Deming, Charbonneau, \& Harrington 2004).

In this Letter, we present the first quantitative model that accounts for all the observed properties of the system. Our main inspiration was the discovery by Johnson \& Winn (2004, hereafter JW04) that in 1970, the eclipses appeared to be diluted by the light of a second star. Building on the demonstration by Herbst et al. (2002) that the ingress and egress light curves can be reproduced by a knife edge crossing the face of a star, we show that the entire historical and modern light curve can be reproduced by a knife edge crossing the mutual orbit of a pair of pre-main-sequence stars. A similar model has been proposed for the central object of planetary nebula NGC 2346, in which a binary system was gradually occulted by an intervening cloud (Schaefer 1985; Méndez et al. 1985; Roth et al. 1984).

In $\S 2$ we review the peculiar phenomenology of $\mathrm{KH} 15 \mathrm{D}$ and describe how it emerges naturally from the model. In $\S 3$ we determine quantitative fits to the data. Finally, in $\S 4$ we

\footnotetext{
${ }^{1}$ Harvard-Smithsonian Center for Astrophysics, 60 Garden Street, Cambridge, MA 02138.

${ }^{2}$ National Science Foundation Astronomy and Astrophysics Postdoctoral Fellow.

${ }^{3}$ Department of Astronomy, University of California at Berkeley, 601 Campbell Hall, Berkeley, CA 94720-3411.

${ }^{4}$ Department of Physics, University of Notre Dame, 225 Nieuwland Science Hall, Notre Dame, IN 46556.
}

discuss the physical interpretation of the model and predict the results of future investigations into this intriguing system.

\section{QUALITATIVE DESCRIPTION OF THE MODEL}

We would like to understand the following characteristics of KH 15D. Every $48.35 \pm 0.02$ days, it decreases in brightness from $I=14.47$ to $\approx 18$ (Hamilton et al. 2001, hereafter H01; Herbst et al. 2002, hereafter H02). Currently, the faint state ("eclipse") lasts for approximately half of the photometric period, and the eclipse duration is increasing by about 1 day $\mathrm{yr}^{-1}$ (H02). During eclipses, the flux has been observed to rise and fall abruptly. In 1995-1996, these "rebrightenings" briefly returned the system to its uneclipsed flux or even brighter. Since 1997, the maximum flux during these events has decreased monotonically ( $\mathrm{H} 01 ; \mathrm{H} 02$ ).

Archival observations from 1913 to 1950 show that the system spent $\$ 20 \%$ of the time greater than 1 mag fainter than its modern bright state (Winn et al. 2003). Between 1967 and 1982, the system alternated from bright to faint with the same period as observed today, but the fractional variation was smaller $(\Delta I=0.67 \pm 0.07)$ and the bright state was $0.90 \pm$ 0.15 mag brighter (JW04). There appears to be a phase shift of $\approx 180^{\circ}$ between the 1967-1982 light curve and the modern light curve; i.e., the modern bright states have nearly the same phase as the previous faint states (JW04).

All these properties are straightforward consequences of the following model. Consider two stars, A and B, with a projected orbit depicted in Figure 1. An opaque screen with a sharp edge oriented vertically (along the $y$-axis) gradually covers the system, moving from left to right (increasing $x$ ). Whenever the orbital motion of a star carries it to the left of the edge $\left(x_{\text {star }}<x_{\text {screen }}\right)$, the starlight is blocked.

Before 1960, both stellar orbits were fully exposed and no eclipses were seen, in compliance with the archival observations. In 1970, the screen covered the left end of B's orbit, but A remained unobscured. As a result, diluted eclipses of star B were observed. By 2002, the screen covered B's entire orbit and a significant fraction of A's orbit, causing today's periodic, long-lasting total eclipses. The eclipses grow in duration as the screen continues to advance. The rebrightenings occurred during the time span around 1995, when B's orbit had not yet been completely covered, allowing B to peek out briefly from behind the screen while A was eclipsed. Because the eclipses in 1970 were of star B, whereas the modern eclipses are of 


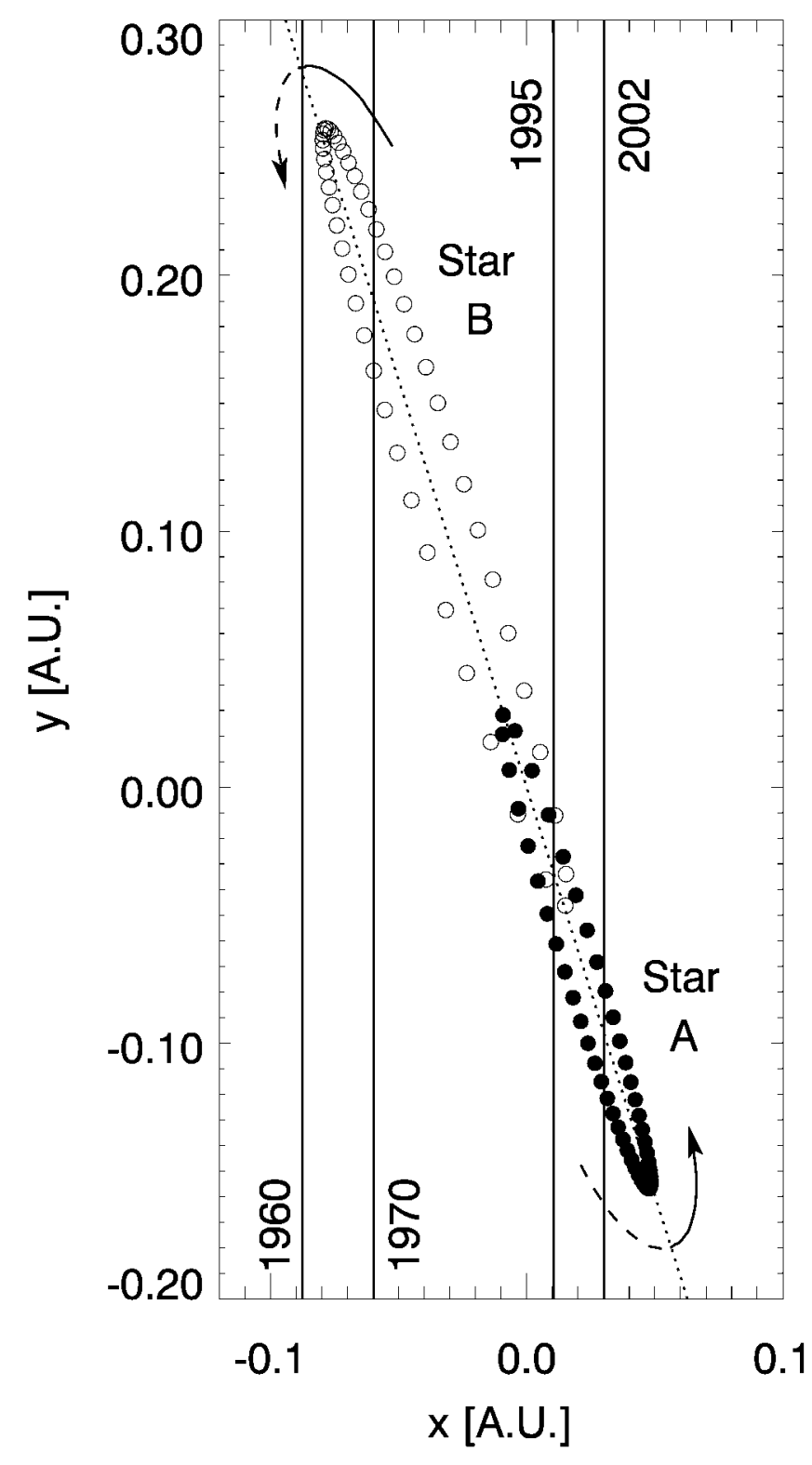

FIG. 1.-Projected binary orbit of model 1 . The center of mass defines the origin, and the $z$-axis points toward the observer. The positions of stars A and $\mathrm{B}$ are plotted with a time sampling $P / 50$. The dashed line is the line of nodes, and the arrows show the motion of the stars between $z>0$ (solid) and $z<0$ (dashed). Vertical lines indicate the edge of the obscuring screen at some years of interest. The screen moves from left to right, blocking the stars whenever they are to the left of the edge.

star A, this model produces the phase shift that was tentatively identified by JW04.

\section{QUANTITATIVE DETERMINATION OF PARAMETERS}

Rather than fitting the model to the voluminous photometric data, we attempted to match some key derived quantities: the eclipse duration in 1967-1970, the eclipse duration and its rate of increase from 1997 to 2003, and the ingress and egress durations of 2002 (see Table 1). We also fitted the radial velocity difference of $3.3 \pm 0.6 \mathrm{~km} \mathrm{~s}^{-1}$ that Hamilton et al. (2003) measured near the start and end of a particular eclipse in 2001.

We assumed that the orbital period is $P=48.35$ days and that star A has mass $0.6 M_{\odot}$ and radius $1.3 R_{\odot}(\mathrm{H} 01)$. The orbit was specified by the mass ratio, eccentricity, and sequential ro-
TABLE 1

Model Constraints

\begin{tabular}{|c|c|c|c|}
\hline Quantity & Star & Year & Value \\
\hline \multirow[t]{6}{*}{ Eclipse fraction.....} & $\mathrm{B}$ & 1968.0 & $0.397 \pm 0.060$ \\
\hline & A & 1997.0 & $0.288 \pm 0.038$ \\
\hline & A & 1999.0 & $0.317 \pm 0.011$ \\
\hline & A & 2000.0 & $0.344 \pm 0.008$ \\
\hline & A & 2002.0 & $0.420 \pm 0.008$ \\
\hline & A & 2003.0 & $0.482 \pm 0.012$ \\
\hline Ingress duration ...... & A & 2002.0 & $3.8 \pm 0.3$ days \\
\hline Egress duration $\ldots . .$. & A & 2002.0 & $5.2 \pm 0.3$ days \\
\hline
\end{tabular}

Notes. - All quantities and their uncertainties were estimated with pencil and ruler from the light curves of $\mathrm{H} 02$, JW04, and P. Garnavich (2003, unpublished). The eclipse fraction is the fraction of the photometric period during which the total flux is below $52 \%$ of the maximum. The ingress and egress durations were estimated by measuring the time between the $I=14.6$ and 17.6 levels and then multiplying by 1.33 to match our choice of limb-darkening model.

tations $\theta_{z}, \theta_{y}$, and $\theta_{x}$ about the Cartesian axes defined in Figure 1. By using Cartesian axes rather than traditional orbital elements, we could capitalize on certain degeneracies of the model. Rotation about the $x$-axis does not affect the light curve. Rotation about the $y$-axis affects the projected size of the orbits but not the projected size of the (spherical) stellar surfaces. Thus, $\theta_{y}$ controls the timescale of changes in eclipse duration relative to the timescales of partial-eclipse phenomena: ingress, egress, and the suppression of rebrightening events.

For a given orbit, we produced a model light curve as follows. The speed of the occulting edge relative to the center of mass was determined by requiring the edge to cross B's orbit between 1963 and 1997. (The actual starting year of the eclipses is unknown.) We computed the positions of the screen and stars with a time sampling of $P / 100$. We assumed $L_{\mathrm{B}} / L_{\mathrm{A}}=1.3$ (the best fit to the JW04 light curve) and added a time-independent flux of $0.04 L_{\mathrm{A}}$ to represent scattered light. Whenever $x_{\text {star }}<$ $x_{\text {screen }}-R_{\text {star }}$, that star made no contribution to the light curve. When the screen covered only part of a stellar surface, we used the same limb-darkening law as $\mathrm{H} 02(\mu=0.3)$ to compute the flux from that star.

First, we optimized $M_{\mathrm{B}}, e$, and $\theta_{z}$ by fitting the eclipse durations and the ratio of durations of ingress and egress. We searched a three-dimensional grid of models with $0.5<M_{\mathrm{B}} / M_{\mathrm{A}}<2.0$, $0.05<e<0.95$, and $-90^{\circ}<\theta_{z}<90^{\circ}$. The best-fitting solution had $\chi^{2} / N_{\text {dof }}=0.7$. Next, we adjusted $\theta_{y}$ to increase the ingress and egress durations to the observed values. Because of symmetry, there were two solutions, differing only in sign. We refer to these as model 1 and model 2. For each model, we forced agreement with the radial velocity measurements by tuning $\theta_{x}$ and the heliocentric radial velocity of the center of mass. Finally, the radius of star $\mathrm{B}$ was tailored to match the ingress and egress durations in 1967-1970, and the timescale over which the rebrightening events decreased in intensity.

The parameters of models 1 and 2, after translating into traditional orbital elements, are given in Table 2. These models produce identical light curves but have different threedimensional orbits and radial velocity curves. (Two additional solutions are obtained by reflecting models 1 and 2 in the $x-z$ plane.) The orbit of model 1 is depicted in Figure 1. Figure 2 shows the time evolution of eclipse durations and ingress/egress ratio. Figure 3 compares the model to the archival light curves. The agreement is excellent within the overall uncertainties in zero point and phase of the archival data. Figure 4 shows the disappearance of the rebrightenings between 1995 and 1998 and 
TABLE 2

Optimized Model Parameters

\begin{tabular}{|c|c|c|}
\hline Parameter & Model 1 & Model 2 \\
\hline Mass ratio, $M_{\mathrm{B}} / M_{\mathrm{A}} \ldots \ldots \ldots \ldots$ & 0.61 & 0.61 \\
\hline Radius of star B (solar radii). & 1.8 & 1.8 \\
\hline Eccentricity, $e \ldots \ldots \ldots \ldots \ldots \ldots \ldots \ldots$ & 0.70 & 0.70 \\
\hline Longitude of ascending node, ${ }^{\mathrm{a}} \Omega(\mathrm{deg})$ & -72.6 & 105.9 \\
\hline Argument of pericenter, $\omega(\mathrm{deg}) \ldots . .$. & -7.2 & 184.9 \\
\hline 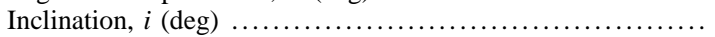 & 84.6 & 81.0 \\
\hline Heliocentric radial velocity of center of $\operatorname{mass}^{\mathrm{b}}\left(\mathrm{km} \mathrm{s}^{-1}\right) \ldots \ldots$ & +15.5 & +5.7 \\
\hline Relative velocity of screen and center of mass $\left(\mathrm{m} \mathrm{s}^{-1}\right) \ldots \ldots \ldots$ & 13 & 13 \\
\hline
\end{tabular}

${ }^{\text {a }}$ Measured counterclockwise, relative to the $x$-axis, as in Fig. 1. The orientation of the occulting edge in celestial coordinates is unknown.

${ }^{\mathrm{b}}$ Positive velocity corresponds to motion away from the Sun.

compares the model with the 2001-2002 light curve. Figure 5 shows the two possible solutions for the radial velocity curve.

\section{DISCUSSION}

We have shown that it is possible to find reasonable stellar and orbital parameters that bring the model into quantitative agreement with the modern and archival light curves and the available radial velocity data. Certainly we do not claim that our model parameters are exactly correct. The fitted parameters are subject to our assumptions about the mass and radius of star A, the year the eclipses began, and the constancy of the screen speed, among other things. Nevertheless, we can use the generic properties of the model to make some inferences and predictions about the system.

The speed of the screen relative to the center of mass of the binary is only $v_{x}=13 \mathrm{~m} \mathrm{~s}^{-1}$, a scale set by the $\approx 35 \mathrm{yr}$ crossing time of star B's projected orbit. This speed seems too small for the screen to be an unrelated foreground cloud. Rather, the screen is probably physically associated with the binary, as also suggested by the small angle between the screen's edge and the orbital plane of the binary. A circular orbit with speed $13 \mathrm{~m} \mathrm{~s}^{-1}$ around a total mass of $1.0 M_{\odot}$ would have a radius of $25 \mathrm{pc}$, which is too large to remain bound to the system. The speed must represent a phenomenon slower than orbital motion, such as orbital precession.

Hence our hypothesis is that the screen is a precessing cir-

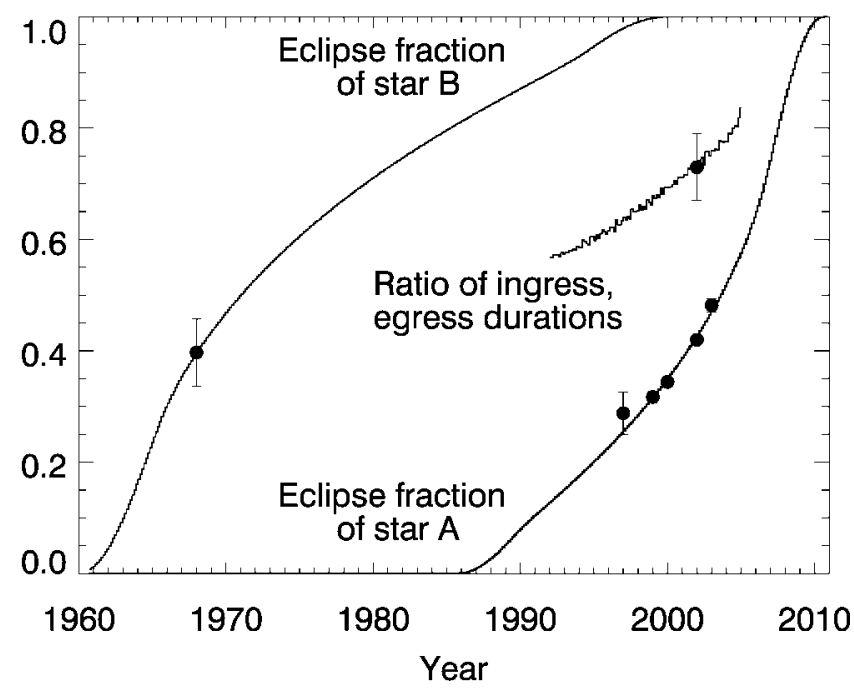

FIG. 2.-Time evolution of the eclipse fractions of stars A and B and the ratio of ingress and egress durations of star A for the best-fit model. The filled circles are observed values (see Table 1). cumbinary disk. As a feasibility test, we numerically integrated the motion of test particles in a circular circumbinary orbit around the binary system of model 1 . For orbits of radius 2.6 AU that are inclined by $\approx 20^{\circ}$ relative to the plane of the binary, the line of nodes regresses at $6.3 \times 10^{-3} \mathrm{rad} \mathrm{yr}^{-1}$, corresponding to a projected velocity of $14 \mathrm{~m} \mathrm{~s}^{-1}$ normal to the plane of the binary. Furthermore, given the mass ratio and eccentricity of the binary, test particles at 2.6 AU are long-term dynamically stable against ejection (Holman \& Wiegert 1999).

One difference between the model and the 2001-2002 light

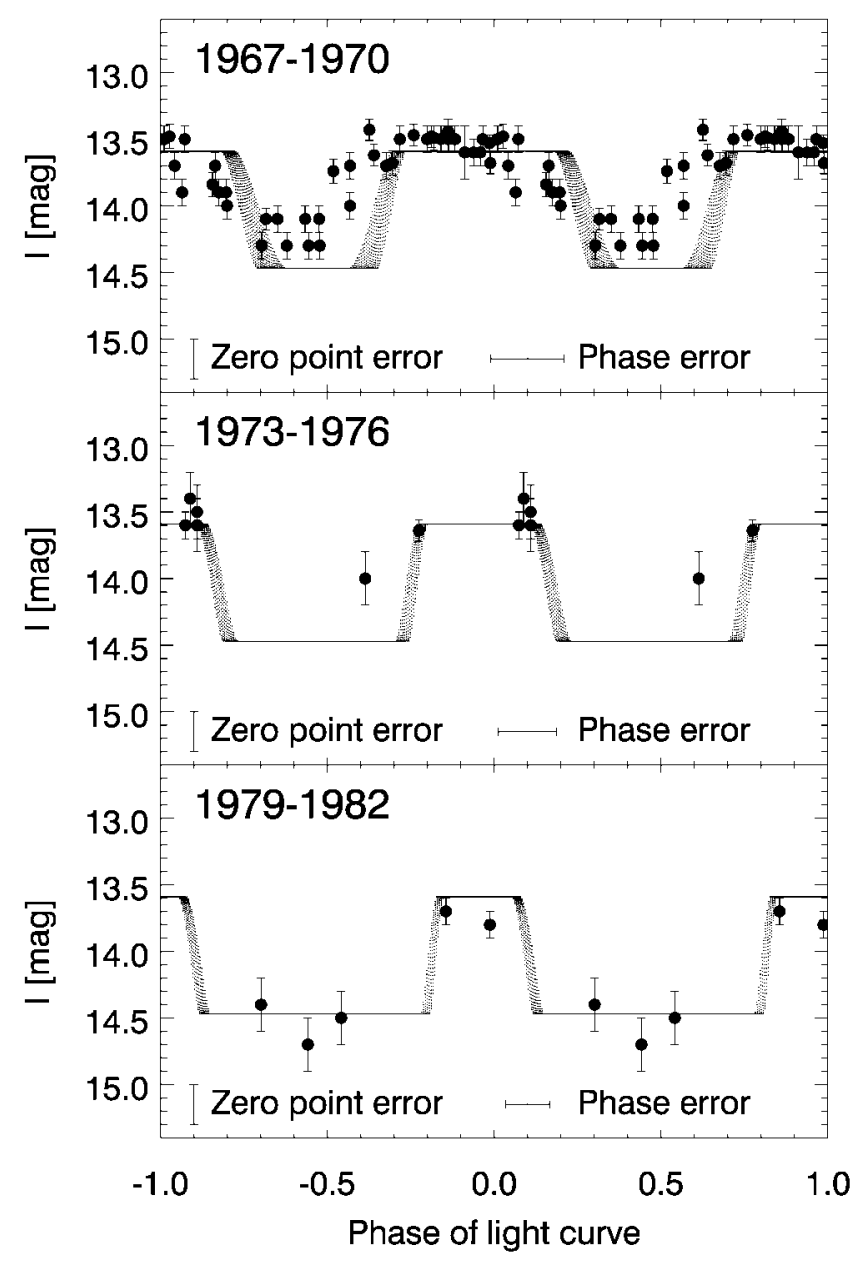

FIG. 3.-Comparison of phased light curves from the model (dotted lines) and data (filled circles) from the Asiago Observatory archive (Barbieri, Omizzolo, \& Rampazzi 2003). The light curves were phased with the H02 ephemeris. The thickness of the model light curves during ingress and egress is due to the growth in eclipse duration during the $3 \mathrm{yr}$ time spans. 

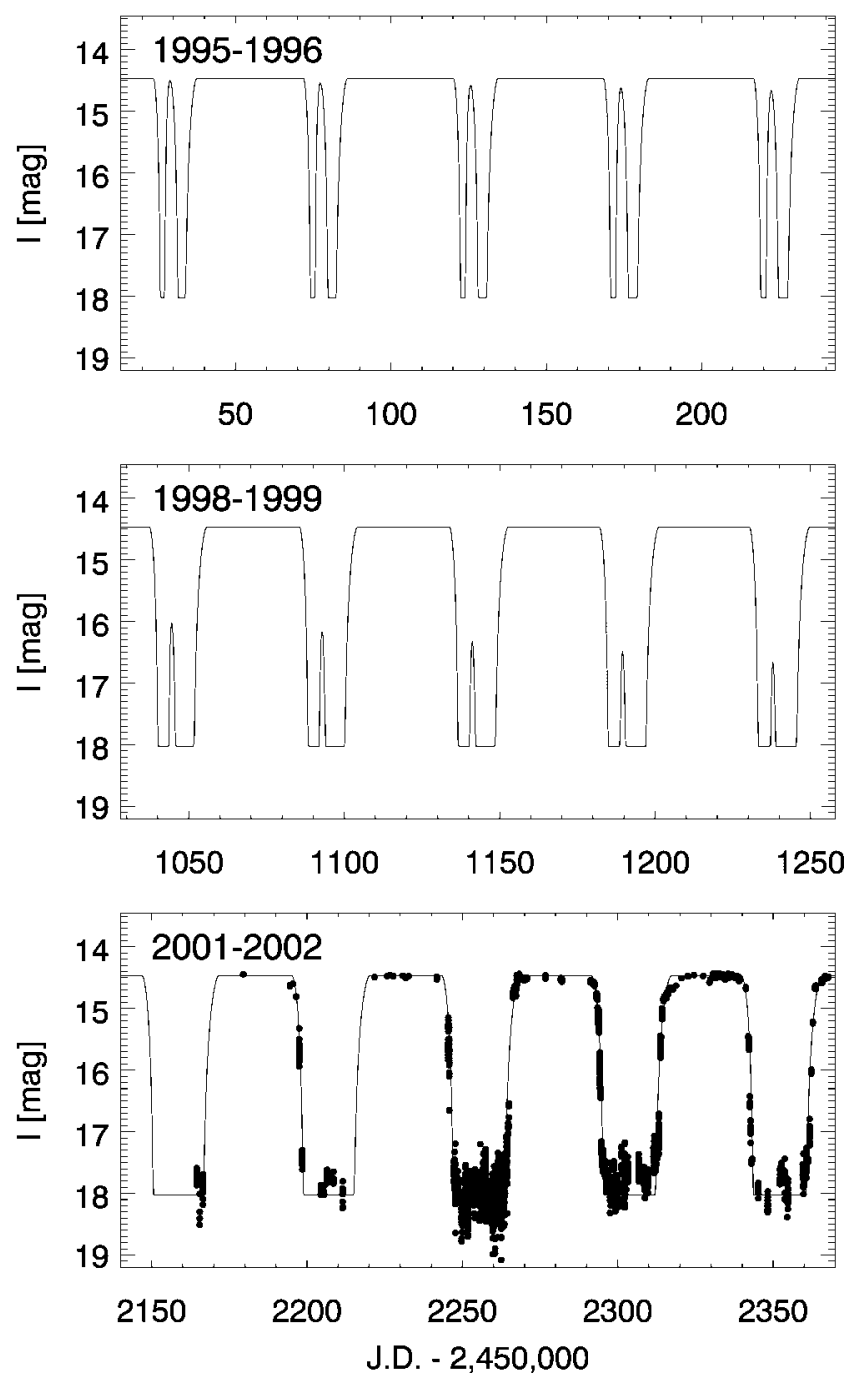

FIG. 4.-Model light curves from three recent time periods. The top and middle panels show the gradual diminution of the amplitude of the rebrightening events. The bottom panel compares the model and the $\mathrm{H} 02$ data.

curve is that the start of ingress and end of egress are too abrupt in the model. This is probably due to the assumption of a perfectly sharp and straight edge. Likewise, we did not attempt to model temporal variations in the scattered light during eclipses, which could be due to the spatial distribution of dust in the plane of the binary or ahead of the occulting edge.

Radial velocity predictions are given in Figure 5, subject to the uncertainty in the total stellar mass and possible contamination of

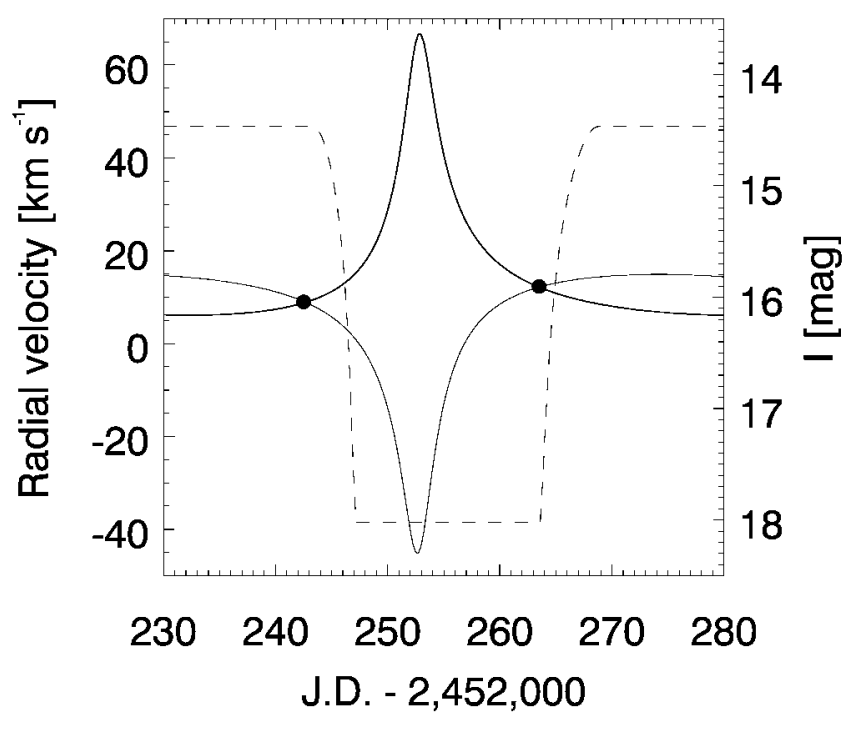

FIG. 5.-Two possibilities for the radial velocity variation of star A (solid lines, left-hand axis). Positive velocity corresponds to motion away from the Sun. Model 1 corresponds to the positive radial velocity peak, and model 2 corresponds to the negative peak. Also plotted is the corresponding I magnitude of the model light curve (dashed line, right-hand axis). Filled circles are measurements by Hamilton et el. (2003).

the H02 measurements by scattered light. Soderblom et al. (1999) found a median radial velocity of $+21.2 \pm 1.8 \mathrm{~km} \mathrm{~s}^{-1}$ for 18 probable members of NGC 2264, favoring model $1(+15.5$ $\left.\mathrm{km} \mathrm{s}^{-1}\right)$ over model $2\left(+5.7 \mathrm{~km} \mathrm{~s}^{-1}\right)$. In either case, the peak-topeak variations outside of eclipses should be $\approx 10 \mathrm{~km} \mathrm{~s}^{-1}$. Larger variations occur during eclipses, when the star's photosphere is hidden.

We can also predict the results of future archival studies and monitoring campaigns. Prior to about 1960, no photometric variations were seen, apart from any intrinsic variations of the stars. The eclipse duration grew between 1970 and 1985, at which point the light curve became more complex because of eclipses of both stars. In the future the eclipse duration will continue to grow, and by about 2012 the system will be completely covered. When the system will come back into view depends on the unknown extent of the obscuring screen.

We are very grateful to Cesare Barbieri, Scott Gaudi, Catrina Hamilton, Bill Herbst, Marc Kuchner, Lucas Laursen, Francesca Rampazzi, Dimitar Sasselov, Brad Schaefer, and Martin Winn for helpful discussions and assistance. This work was supported by the National Science Foundation under grant 0104347.

\section{REFERENCES}

Agol, E., Barth, A. J., Wolf, S., \& Charbonneau, D. 2004, ApJ, 600, 781

Barbieri, C., Omizzolo, A., \& Rampazzi, F. 2003, Mem. Soc. Astron. Italiana, 74, 430

Barge, P., \& Viton, M. 2003, ApJ, 593, L117

Deming, D., Charbonneau, D., \& Harrington, J. 2004, ApJ, 601, L87

Grinin, V. P., \& Tambovtseva, L. V. 2002, Astron. Lett., 28, 601

Hamilton, C. M., Herbst, W., Mundt, R., Bailer-Jones, C. A. L., \& JohnsKrull, C. M. 2003, ApJ, 591, L45

Hamilton, C. M., Herbst, W., Shih, C., \& Ferro, A. J. 2001, ApJ, 554, L201 (H01)

Herbst, W., et al. 2002, PASP, 114, 1167 (H02)

Holman, M., \& Wiegert, C. 1999, AJ, 117, 621
Johnson, J., \& Winn, J. N. 2004, AJ, in press (astro-ph/0312428) (JW04)

Kearns, K. E., \& Herbst, W. H. 1998, AJ, 116, 261

Méndez, R. H., Marino, B. F., Clariá, J. J., \& van Driel, W. 1985, Rev. Mexicana Astron. Astrofis., 10, 187

Roth, M., Echevarría, J., Tapia, M., Carrasco, L., Costero, R., \& Rodríguez, L. F. 1984, A\&A, 137, L9

Schaefer, B. E. 1985, ApJ, 297, 245

Soderblom, D. R., King, J. R., Siess, L., Jones, B. F., \& Fischer, D. 1999, AJ, 118,1301

Winn, J. N., Garnavich, P. M., Stanek, K. Z., \& Sasselov, D. D. 2003, ApJ, 593, L121 